\title{
Non-Markovian theory of open systems in classical limit
}

\author{
A. A. Neufeld ${ }^{\text {a) }}$ \\ Abteilung für Spektroskopie und Photochemische Kinetik, Max-Planck-Institut für Biophysikalische Chemie, \\ Am Fassberg, D-37077 Göttingen, Germany
}

(Received 2 February 2004; accepted 14 May 2004)

\begin{abstract}
A fully classical limit of the recently published quantum-classical approximation [A. A. Neufeld, J. Chem. Phys. 119, 2488 (2003)] is obtained and analyzed. The resulting kinetic equations are capable of describing the evolution of an open system on the entire time axis, including the short-time non-Markovian stage, and are valid beyond linear response regime. We have shown, that proceeding to the classical mechanics limit we restrict the class of allowed correlations between an open system and a canonical bath, so that the initial conditions and the relaxation operator has to be appropriately modified (projected). Disregard of the projection may lead to unphysical behavior, since mechanism of the decay of some correlations is essentially of quantum-mechanical nature, and is not correctly described by classical mechanics. The projection (quantum correction to the kinetics) is particularly important for the non-Markovian regime of relaxation towards canonical equilibrium. The conformity of the developed method to the conventional approaches is demonstrated using a model of Brownian motion (heavy particle in the bath of light ones), for which the obtained non-Markovian equations are reduced to the standard Fokker-Planck equation in phase space.

(C) 2004 American Institute of Physics. [DOI: 10.1063/1.1769353]
\end{abstract}

\section{INTRODUCTION}

The concept of open system (OS) plays an important role in the consideration of complex many-body dynamics. In many situations one can define OS as a relatively small subsystem of interest, weakly coupled to the remaining part of the system (bath). In this case interactions between the OS and the bath can be accounted for by means of perturbation theory, which appreciably facilitates an analysis of the corresponding dynamical properties. The meaning of weak coupling alters in accordance with the theoretical approach in use, while the so-called linear response theory ${ }^{1-3}$ is most successful to present days as it provides an unified treatment of the kinetics at small deviations from the equilibrium. However, processes of quantum nature and/or far from equilibrium require different kind of approaches, which stimulated the development of alternative treatments.

Direct solution of Newton's equations for many-body system with a finite, but large number of constituents, as a prototype of a system in the thermodynamic limit, forms the basis of the nonequilibrium molecular dynamics (NEMD) methods, ${ }^{4}$ and allows for nonperturbative simulations within the frameworks of classical mechanics. Considerable efforts were put to add effects of quantum transitions directly to the classical trajectories. ${ }^{5-10}$ There are, however, some fundamental problems in this type of approaches. In particular, equations of classical mechanics are deterministic, while quantum subsystems are intrinsically probabilistic. As a result, the deterministic feedback of quantum subsystem on a classical trajectory, necessary to propagate classical degrees of freedom, can be obtained just in two ways, either invoking a mean-field approximation, ${ }^{5-7,10}$ or using a surface hopping

${ }^{\text {a)} E l e c t r o n i c ~ a d d r e s s: ~ a n e u f e l @ g w d g . d e ~}$ scheme. $^{7-10}$ The drawbacks of these approaches are well known, mean-field approximation fails to describe properly the situation of considerably distinct exit channels of reaction, while surface hopping is an ad hoc scheme with unknown accuracy and applicability limits.

Quantum-classical (QC) approaches, which treat both quantum and classical subsystems on a similar probabilistic footing, are better balanced and do not suffer from the aforementioned problems. There exist two general schemes for the derivation of kinetic equations for the reduced density matrix (RDM) of quantum subsystem. One scheme relies upon partial Wigner transform ${ }^{11,12}$ over classical degrees of freedom and yields the quantum-classical Liouville equation $^{13-17}$ (QCLE), while the others approaches ${ }^{18-25}$ start with an equivalent integrodifferential representation of the quantum Liouville-von Neumann equation.

The QCLE is probably the simplest quantum-classical theory for the RDM of quantum subsystem. Its appealing feature is a formal similarity both to the Liouville-von Neumann equation (for quantum subsystem) and to the Liouville equation for the reduced distribution function (for classical degrees of freedom). Mathematical background of QCLE is a formal expansion of partial Wigner transform in terms of $\hbar$ $\rightarrow 0$ with the subsequent replacement of quantum operators (positions and momenta) in Poisson brackets with their classical counterparts (phase space coordinates). However, the last step is rather suspicious, and cannot be justified. In particular, one can point out two associated defects of QCLE. First, substitution of quantum operators by the corresponding phase space coordinates means elimination of many variables (the detailed picture of the distribution over quantum levels is omitted). As it follows from the Nakajima-Zwanzig projection formalism, ${ }^{18}$ any rigorous elimination of variables unavoidably leads to memory-type of equations. The subse- 
quent approximations to a memory kernel may sometimes lead to the differential equations, and the conditions for that (applicability limits) are also obtained at this step. This stage is apparently missed in the derivation of QCLE, which is immediately obtained in the differential form. As a result, the applicability limits of QCLE are unknown.

Another drawback of QCLE is more substantial. The replacement of quantum operators by the phase space coordinates in the framework of partial Wigner transforms of the quantum Liouville-von Neumann equation also leads to the replacement of true cross coherences, which drive the energy flow between the subsystems, by direct coherences inside quantum subsystem, as it was well explained in Ref. 26 on the basis of a simple four-level system. This defect is inherent to all QC treatments, which model the true interactions between subsystems by a time-dependent external field within the framework of the Schrödinger or Liouville equation.

Recently, a novel non-Markovian quantum-classical approximation $^{25}$ (NQCA) was suggested, which does not suffer from the aforementioned drawbacks peculiar to QCLE. The approach is based on a short coherence time of cross correlations between the quantum subsystem and the canonical bath, due to an energy dispersion of the bath degrees of freedom. It is of differential form, but fully accounts for arbitrary long memory of the bath. NQCA also has known applicability limits, and properly handles cross correlations between quantum and classical subsystems, including an initial time period, when a differential description is not valid. On the other hand, NQCA is quite different from QCLE, and is not reduced to the Liouville equation for the partial distribution function in the full classical limit, i.e., when the subsystem of interest (which is treated quantummechanically within QC approaches) is also described by means of classical mechanics.

In the present paper we obtain and analyze the full classical limit of NQCA to clarify the physical meaning of basic ingredients of the theory in the domain of classical mechanics. The outline of the paper is following: Basic equations of NQCA are reproduced in Sec. II, and serve as a starting point for the subsequent consideration. The full classical limit is obtained in Sec. III using a standard procedure. Basic properties of the resulting kinetic equations (applicability criterion, direct incorporation of the canonical equilibrium), and a physical origin of projected dynamics (quantum corrections) are discussed ibidem. The latter prevents from the violation of positivity of the reduced distribution function within the applicability limits of the approach, as it is explicitly demonstrated in Sec. IV using a simple model of harmonic oscillator bilinearly coupled to a single correlation time canonical bath. The conformity of the obtained non-Markovian kinetic equations with the traditional approaches is revealed for a Brownian dynamics model (heavy particle in the bath of light ones), for which in the Markovian limit one gets a standard Fokker-Planck equation in phase space, see Sec. V and Appendix A. Extended capabilities of the approach and importance of quantum corrections for a short-time dynamics are demonstrated in Sec. VI considering non-Markovian relaxation in the momentum space. A simple method of deriv- ing the Smoluchowski equation (diffusion in position space) starting directly from the Fokker-Planck treatment is outlined in Sec. VII and Appendix B. The results are briefly summarized in the Conclusion.

\section{BASIC EQUATIONS OF NON-MARKOVIAN QUANTUM-CLASSICAL APPROXIMATION}

A quantum-classical approximation ${ }^{25}$ requires matrix elements of the system-bath coupling $\hat{W}(q)$ to satisfy the condition

$$
\frac{\left|W_{i k}(q)\right|}{\hbar} \tau_{b} \ll 1,
$$

where $\hbar$ is the Planck constant, $q$ denotes a generalized set of the phase space coordinates of the bath, and the characteristic lifetime $\tau_{b}$ of cross correlations between open quantum subsystem and heat reservoir is

$$
\tau_{b}=\frac{\hbar}{\pi k_{B} T} \text {. }
$$

In order to reduce the number of notations we set the Boltzmann constant $k_{B}=1$, thus measuring absolute temperature $T$ in energy units. At times $t \gg \tau_{b}$ NQCA reduces to the set of differential equations ${ }^{25}$

$$
\begin{aligned}
\frac{d \sigma(t)}{d t}=- & \frac{i}{\hbar}[\hat{H}, \sigma(t)]-\frac{i}{\hbar} \int[\hat{W}(q), \eta(q ; t)] d q \\
\frac{\partial \eta(q ; t)}{\partial t}=- & \frac{i}{\hbar}[\hat{H}, \eta(q ; t)]-\frac{i}{\hbar}[\hat{W}(q), \sigma(t)] \\
& +\frac{1}{\hbar}[\hat{U}(q), \sigma(t)]_{+}+\frac{1}{\hbar}\left[\hat{Y}(q), \frac{d \sigma(t)}{d t}\right. \\
& \left.+\frac{i}{\hbar}[\hat{H}, \sigma(t)]\right]_{+}+\mathcal{L}_{q} \eta(q ; t),
\end{aligned}
$$

where $\hat{H}$ is a Hamiltonian of open system, $\sigma(t)$ and $\eta(q ; t)$ are RDM of quantum subsystem and auxiliary matrix, respectively, while $\mathcal{L}_{q}$ is a linear functional operator defining motion of the bath in its phase space. Here also $[\ldots, \ldots]$ and $[\ldots, \ldots]_{+}$denote commutator and anticommutator, correspondingly,

$$
\hat{U}(q)=\int_{-\infty}^{\infty} f_{b}(\tau) e^{-(i / \hbar) \hat{H} \tau} \hat{W}(q) e^{(i / \hbar) \hat{H} \tau} d \tau,
$$

and

$$
\hat{Y}(q)=-\int_{-\infty}^{\infty} \tau f_{b}(\tau) e^{-(i / \hbar) \hat{H} \tau} \hat{W}(q) e^{(i / \hbar) \hat{H} \tau} d \tau,
$$

where the generalized memory kernel, describing the decay of cross correlations between open quantum subsystem and thermal bath, and its Fourier transform are

$$
f_{b}(t)=-\frac{1}{\pi \tau_{b}} \frac{1}{\sinh \left(t / \tau_{b}\right)}, \quad \tilde{f}_{b}(\omega)=i \tanh \left(\frac{\hbar \omega}{2 T}\right) .
$$

The initial conditions for Eqs. (3) and (4) are of the form 


$$
\sigma(t=0)=\sigma_{0}, \quad \eta(q ; t=0)=\frac{1}{\hbar}\left[\hat{Y}(q), \sigma_{0}\right]_{+},
$$

where the nonzero initial conditions for auxiliary matrix account for the correlations created during the initial short-time $\left(t \lesssim \tau_{b}\right)$ evolution of quantum subsystem, see Ref. 25 for more details. To the end of this section we also note, that the approach requires renormalization of the Hamiltonians of the form

$$
\hat{H} \rightarrow \hat{H}+\operatorname{Tr}_{b}\left(\hat{W} \rho_{b}\right), \quad \hat{W} \rightarrow \hat{W}-\operatorname{Tr}_{b}\left(\hat{W} \rho_{b}\right),
$$

to eliminate long lasting source term in the general master equation. Here $\operatorname{Tr}_{b}$ denotes the trace over the bath variables, while $\rho_{b}$ is the equilibrium density matrix of the bath.

\section{THE CLASSICAL LIMIT}

First of all we find the classical limit of operators, defined by Eqs. (5) and (6). Substituting the short-time expansion of the interaction between open system and thermal bath in the Heisenberg representation

$$
e^{-(i / \hbar) \hat{H} \tau} \hat{W}(q) e^{(i / \hbar) \hat{H} \tau} \simeq \hat{W}(q)-\frac{i}{\hbar}[\hat{H}, \hat{W}(q)] \tau+\cdots,
$$

in Eqs. (5) and (6), and taking into account that $f_{b}(\tau)$ decays fast, being an antisymmetric function of time, we get

$$
\hat{U}(q) \simeq \frac{\hbar}{2 T} \frac{i}{\hbar}[\hat{H}, \hat{W}(q)], \quad \hat{Y}(q) \simeq \frac{\hbar}{2 T} \hat{W}(q) .
$$

Then, the classical limit of Eqs. (3) and (4) is obtained as usual, via the standard correspondence relations

$$
\begin{aligned}
& \frac{i}{\hbar}[\hat{H}, \sigma(t)] \rightarrow\{H(Q), f(Q ; t)\}, \\
& {[\hat{H}, \sigma(t)]_{+} \rightarrow 2 H(Q) f(Q ; t),}
\end{aligned}
$$

i.e., the RDM $\sigma(t)$ is replaced by the reduced distribution function (RDF) $f(Q ; t)$, depending on the phase space coordinates of open system $Q$, the auxiliary matrix becomes the auxiliary function, the Hamiltonian operator $\hat{H}$ is substituted by the Hamilton function $H(Q)$, the anticommutator reduces to the product of the corresponding classical analogs, while commutators are replaced by Poisson brackets of the form

$$
\begin{aligned}
\{H(Q), f(Q ; t)\}= & \frac{\partial H(P, X)}{\partial P} \frac{\partial f(P, X ; t)}{\partial X} \\
& -\frac{\partial H(P, X)}{\partial X} \frac{\partial f(P, X ; t)}{\partial P},
\end{aligned}
$$

where $X$ and $P$ denote arbitrary sets of generalized coordinates and canonically conjugate momenta, correspondingly. Application of relations (12) to Eqs. (3), (4), and (11) yields

$$
\begin{aligned}
\frac{\partial f(Q ; t)}{\partial t}= & -\{H(Q), f(Q ; t)\} \\
& -\int\{W(Q, q), \eta(Q, q ; t)\} d q,
\end{aligned}
$$

$$
\begin{aligned}
\frac{\partial \eta(Q, q ; t)}{\partial t}= & -\{H(Q), \eta(Q, q ; t)\}-\{W(Q, q), f(Q ; t)\} \\
& +\frac{1}{T}\{H(Q), W(Q, q)\} f(Q ; t) \\
& +\frac{W(Q, q)}{T}\left(\frac{\partial f(Q ; t)}{\partial t}+\{H(Q), f(Q ; t)\}\right) \\
& +\mathcal{L}_{q} \eta(Q, q ; t),
\end{aligned}
$$

with the initial conditions

$$
f(Q ; t=0)=f_{0}(Q), \quad \eta(Q, q ; t=0)=\frac{W(Q, q)}{T} f_{0}(Q),
$$

readily obtained from Eq. (8). The canonical form of Eqs. (14) and (15) allows to obtain non-Markovian kinetic equations for the arbitrary set of phase space coordinates immediately, since Poisson brackets (13) are invariant with respect to canonical transformations. In particular, the use of the customary coordinates and momenta is convenient at high friction, while the action-angle variables are preferable in the low friction limit (energy diffusion regime).

The obtained kinetic equations of the full classical limit differ from the existing approaches, ${ }^{19,27-29}$ based on the stochastic models. Specifically, the generalized Langevin equation (GLE) is an equation for the particular realization of the driving stochastic process. The statistical properties of the thermal reservoir enter the theory when the obtained solution is averaged over the initial states of the bath, sampled from the corresponding distribution. As a result, the GLE does not explicitely involve the temperature of the bath, but incorporates it via a proper relation between the autocorrelation function of the fluctuating external force and the memory (friction) kernel. The required connection is given by famous fluctuation-dissipation theorem (FDT), which provides a link between fluctuations and the linear response.

We employed a considerably different approach. In particular, the small parameter of the theory is directly related to the statistical properties of the heat reservoir, and is independent of the bath dynamics. In other words, the GLE is founded on the dynamical properties of the system under consideration, while our approach is based on the statistical properties of the bath, and is valid beyond the linear response regime. As the result, our equations incorporate the canonical equilibrium directly, and the FDT is not required at all. Indeed, the stationary solution of Eqs. (14) and (15) is determined from the conditions $\partial_{t} f(Q, q ; t)=0$ and $\eta(Q, q ; t)$ $=0$, which gives $\left[f_{\infty}(Q)=\lim _{t \rightarrow \infty} f(Q ; t)\right]$

$$
\begin{aligned}
& \left\{H(Q), f_{\infty}(Q)\right\}=0, \\
& \left\{W(Q, q), f_{\infty}(Q)\right\}=\frac{1}{T}\{H(Q), W(Q, q)\} f_{\infty}(Q) .
\end{aligned}
$$

It is readily seen, that the static contour

$$
f_{\infty}(Q)=e^{-H(Q) / T}
$$


satisfies both conditions (17), which is proved directly by using Eq. (13), and is the stationary solution of the kinetic Eqs. (14) and (15).

The transition from the quantum-classical to the purely classical treatment of an open system deserves additional discussion. The characteristic time $\tau_{b}$ of thermal fluctuations (2) plays the central role in our quantum-classical treatment. ${ }^{25}$ However, this characteristic time does not exist in the classical limit, since $\hbar \rightarrow 0$ leads to $\tau_{b} \rightarrow 0$ as well. None the less, specific correlations between open system and thermal bath, which are formed on the time scale of $\tau_{b}$, survive in the classical limit, see Eq. (16), for instance. Mathematically this fact is explained by a close look on Eq. (5). It is readily seen, that at $\hbar \rightarrow 0$ the memory kernel $f_{b}(\tau)$ decays instantaneously, but, at the same time, exponentials in the integrand oscillate infinitely fast as well. The resulting value is actually independent on $\hbar$, see the first of Eqs. (11). The small parameter (1) can also be written in the form

$$
\frac{|W(Q, q)|}{\pi T} \ll 1,
$$

which is independent on $\hbar$. In particular, Eq. (19) means, that one can neglect the influence of the interaction between the open system and the bath on equilibrium, otherwise the open system would relax to an equilibrium state, which is different from that given by Eq. (18) and were dependent on the system-bath coupling.

In statistical physics based on classical mechanics, the Liouville equation for the distribution function of the total system in its phase space usually serves as the starting point for the derivation of various kinetic equations. The associated problem of a proper choice of the initial conditions is well known, a many-body system has an infinite number (in the thermodynamic limit) of "pathologic" initial states (or correlations), for which the evolution of the system is not ergodic at all. Although the probability of realization of such initial states is negligibly small, the very existence of them produces considerable mathematical problems in statistical mechanics. The reduced description of many-body system in terms of kinetic equations is valid only for specific initial correlations, as it was clearly demonstrated by the concept of subdynamics. ${ }^{30}$

In our case, however, the physical origin of the modified (projected) initial conditions seems to be different. Accordingly to the picture, provided by NQCA,${ }^{25}$ some of the cross correlations between open system and the bath decay on the time scale of $\tau_{b}$, which vanishes when passing to the limit of classical mechanics. (The uncorrelated state is actually a specific superposition of all correlation patterns ${ }^{30}$ by analogy with the resolution of identity, so that the formation of correlations may alternatively be viewed as the decay of some of them.) In other words, the mechanism of their decay is of quantum-mechanical nature, and can not be properly described classically. Exactly such correlations are eliminated from the initial state by setting the corresponding nonzero initial conditions for the auxiliary function $\eta$, see Eq. (16).

Within the framework of NQCA, ${ }^{25}$ the modified initial conditions were obtained in a systematic way, through the expansion of the non-Markovian memory kernel to next or- ders using the fast decay of $f_{b}(t)$. Such a procedure leads not only to a change of the initial conditions, but modifies the relaxation operator as well. In other words, Eq. (15), which is responsible for a proper treatment of correlations between open system and thermal bath, also accounts for a nonclassical decay of some correlations in the course of relaxation to equilibrium, thus leading to the quantum corrections to the kinetics. [The corresponding term contains a time derivative on the right-hand side of Eq. (15).] A close look at Eqs. (14) and (15) shows, that the quantum corrections do not appear in the dynamics of the isolated subsystem $[W(Q, q)=0]$, and at thermal equilibrium. This means, that the obtained nonclassical corrections concern the nonequilibrium energy flow between the subsystems, which appears to have both the classical and the quantum components. The latter is of increasing importance at moderate values of the parameter (19), and may prevail, if the condition (19) is not fulfilled. Nevertheless, the MD methods, which neglect the quantum effects, can be used for the ab initio simulation of equilibrium bath. The nonclassical (or quantum) corrections may substantially influence the kinetics in the non-Markovian regime, see Secs. IV and VI. Another closely related subject is the so-called quantum correction factor in the theory of highfrequency spectra of liquids. ${ }^{31-33}$

To the end of this section we would like to emphasize, that taking the static contour (18) as the initial condition for the open system may lead to transient kinetics, since the initial conditions for the auxiliary function $\eta$ (16) are nonzero in this case. This is because the initial condition for the total system was taken as the uncorrelated product of the states of the open system and of the bath. On the other hand, the true equilibrium state is characterized by the specific correlations not only inside the open system and inside the bath, but also between them. Thus, the transient kinetics in the above case corresponds to the formation of the proper correlations between the subsystems, despite the fact that the stationary solution for the state of open system is the static contour again.

Below we give an example, demonstrating the physical importance of the proper initial correlations (16), on the basis of a simple model of one-dimensional harmonic oscillator bilinearly coupled to a bath.

\section{ROLE OF THE INITIAL CORRELATIONS}

A bilinear coupling is frequently used to model interaction between an open system and a thermal reservoir. Physically it corresponds to the linear expansion of the true system-bath potential, and, therefore, assumes the space restricted motion of open system. One of the simplest models of this type is a one-dimensional harmonic oscillator with the Hamiltonian

$$
H(p, x)=\frac{p^{2}}{2 m}+\frac{1}{2} m \omega_{0}^{2} x^{2},
$$

where $m$ and $\omega_{0}$ are the effective mass and angular frequency of oscillator, respectively. The coupling to the bath is assumed to have a bilinear form, i.e.,

$$
W(Q, q)=x B(q),
$$


while the bath will be characterized by a single correlation time $\tau_{c}$, so that

$$
\mathcal{L}_{q}=-\frac{1}{\tau_{c}},
$$

and

$$
B(q)=B,
$$

which is equivalent to the bath correlation function of the form

$$
\overline{B[q(t)] B[q(0)]}=B^{2} e^{-t / \tau_{c}},
$$

where an overline denotes ensemble average.

The applicability criterion (19) yields for this case the following estimate:

$$
\Lambda^{2}=\frac{B^{2}}{m T \omega_{0}^{2}} \ll \frac{\pi^{2} T}{2 E},
$$

where $E$ is the energy of the oscillator. The relation (24) comes from considering the maximum oscillator displacement as $x_{\max }=\sqrt{2 E /\left(m \omega_{0}^{2}\right)}$. Thus, the applicability limits for the model under consideration actually depend on the state of the open system. For instance, $\Lambda^{2} \ll \pi^{2} / 2$ guarantees the validity of the approach at energies lower or near equilibrium $(E \sim T)$. On the other hand, when using initial conditions with the energies considerably larger than the mean heat energy, condition (24) becomes more restrictive.

In the following we use dimensionless variables, and measure the time, momentum, and displacement in units of $1 / \omega_{0}, \sqrt{m \omega_{0}}$, and $1 / \sqrt{m \omega_{0}}$, respectively. To reduce the number of notations, we denote these dimensionless units by the same symbols. Then, the Hamiltonian (20) takes the simple form

$$
H(p, x)=\frac{1}{2}\left(p^{2}+x^{2}\right) .
$$

The corresponding set of kinetic equations, describing energy relaxation, is readily obtained from Eqs. (14)-(16), (20)-(22), and is (using the dimensionless units)

$$
\begin{aligned}
& \frac{d}{d t}\left\langle x^{2}(t)\right\rangle=2\langle p x(t)\rangle, \\
& \frac{d}{d t}\left\langle p^{2}(t)\right\rangle=-2\langle p x(t)\rangle-2 \mu(t), \\
& \frac{d}{d t}\langle p x(t)\rangle=\left\langle p^{2}(t)\right\rangle-\left\langle x^{2}(t)\right\rangle-\zeta(t),
\end{aligned}
$$

and

$\frac{d \mu(t)}{d t}=-\left(1+\Lambda^{2}\right) \zeta(t)+\Lambda^{2}\left[\left\langle p^{2}(t)\right\rangle-T\right]-\frac{\mu(t)}{\tau_{c}}$,

$\frac{d \zeta(t)}{d t}=\mu(t)+\Lambda^{2}\langle p x(t)\rangle-\frac{\zeta(t)}{\tau_{c}}$

where the parameter $\Lambda^{2}$ was defined in Eq. (24), and the average of any dynamic variable $a(Q)$ is defined as

$$
\langle a(t)\rangle=\int a(Q) f(Q ; t) d Q .
$$

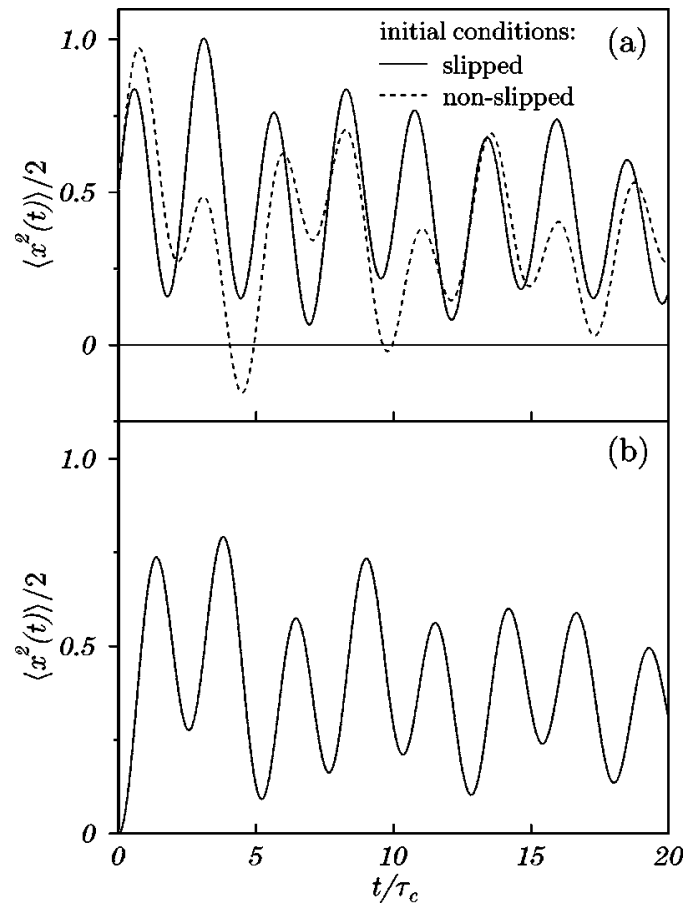

FIG. 1. Evolution of the potential energy of a harmonic oscillator at moderate strength of linear coupling with a bath. The parameters are $\Lambda^{2}=0.5$, $\tau_{c}=10$, and $T=0.5$. In the case (a) (upper panel) the initial conditions were $\left\langle p^{2}\right\rangle_{0}=1,\left\langle x^{2}\right\rangle_{0}=1$, and $\langle p x\rangle_{0}=1$, while the corresponding initial conditions for the auxiliary functions were either taken from Eq. (33) (solid line), or set to zero (dashed line). In the case (b) (lower panel) the initial conditions were $\left\langle p^{2}\right\rangle_{0}=2,\left\langle x^{2}\right\rangle_{0}=0$, and $\langle p x\rangle_{0}=0$, for which Eq. (33) gives zero initial conditions for the auxiliary functions. It is seen, that neglecting the initial correlations one gets unphysical behavior of temporary negative potential energy.

The initial conditions for Eqs. (26)-(30) are

$\left\langle x^{2}(0)\right\rangle=\left\langle x^{2}\right\rangle_{0}, \quad\left\langle p^{2}(0)\right\rangle=\left\langle p^{2}\right\rangle_{0}, \quad\langle p x(0)\rangle=\langle p x\rangle_{0}$,

and

$\mu(0)=\Lambda^{2}\langle p x\rangle_{0}, \quad \zeta(0)=\Lambda^{2}\left\langle x^{2}\right\rangle_{0}$,

where nonzero initial conditions for the auxiliary functions $\mu(t)$ and $\zeta(t)$ account for the initial correlations in the system. The modification of the initial conditions, caused by the evolution during neglected time scales, is frequently called "slippage," and we also use this terminology here. The analytic solution of the kinetic Eqs. (26)-(30) is rather cumbersome, and, therefore, we solve them numerically to demonstrate the physical importance of the slipped initial conditions (33).

Figures 1(a) and 1(b) show the evolution of the potential energy $\left[\left\langle x^{2}(t)\right\rangle / 2\right.$ in the reduced units] at moderate strength of the interaction between the open system and the bath. The parameters used are: $\Lambda^{2}=0.5, \tau_{c}=10$, and $T=0.5$. In the first case (a) the initial conditions were $\left\langle p^{2}\right\rangle_{0}=1,\left\langle x^{2}\right\rangle_{0}=1$, and $\langle p x\rangle_{0}=1$. In the second case (b) they were $\left\langle p^{2}\right\rangle_{0}=2$, $\left\langle x^{2}\right\rangle_{0}=0$, and $\langle p x\rangle_{0}=0$, and the initial energies are equal in both cases. In the first case the initial conditions for the auxiliary functions (33) are nonzero, and their neglect leads to the unphysical result of temporary negative potential energy, which also means positivity violation of partial distribution 
function, see Fig. 1(a). Similar unphysical behavior can be observed for the kinetic energy, if the slippage was neglected. In the second case (b) the initial values for auxiliary functions are naturally zero, but at the same time the kinetics does not exhibit unphysical features, see Fig. 1(b).

Thus, the role of the slipped initial conditions is similar to those in the corresponding quantum-classical treatment, ${ }^{26}$ where they remove the breakdown of the positivity of the RDM within the applicability limits of the approach. In the full classical limit the slippage preserves positivity of the $\mathrm{RDF}$, and is particularly important for the non-Markovian stage in the kinetics and at moderate strength of the systembath interaction, in the sense of the critical parameter (19). On the other hand, methods like NEMD, ${ }^{4}$ which essentially rely upon the appropriate sampling of the initial state, slip away of this problem. Nevertheless, despite the fact that NEMD methods intrinsically preserve positivity of the reduced distribution function at any circumstances, the question about adequacy of the short-time kinetics, obtained by NEMD calculations without taking care of quantum corrections is wide open.

\section{KINETIC EQUATION FOR DELTA-CORRELATED BATH}

The non-Markovian kinetic equations, obtained in Sec. III, provide an accurate description of open systems interacting with a thermal bath in the full classical limit. The approach has wide applicability limits, but must reproduce known results, at least under more restricted conditions. To demonstrate such a conformity we consider the Markovian limit for the model consisting of a particle in an external field, and coupled to the canonical bath. Then, the Hamiltonian of open system takes the form

$$
H(\mathbf{p}, \mathbf{r})=\frac{\mathbf{p}^{2}}{2 m}+u(\mathbf{r}),
$$

where $u(\mathbf{r})$ is the potential of the external field, which is independent on the bath coordinates. In turn, Poisson bracket (13) becomes

$$
\{H(Q), f(Q ; t)\}=\left(\frac{\mathbf{p}}{m} \cdot \nabla_{\mathbf{r}}-\nabla_{\mathbf{r}} u(\mathbf{r}) \cdot \nabla_{\mathbf{p}}\right) f(\mathbf{p}, \mathbf{r} ; t),
$$

where $\boldsymbol{\nabla}_{\mathbf{r}}$ and $\boldsymbol{\nabla}_{\mathbf{p}}$ are gradient operators in position and momentum spaces, respectively, while the centered dot denotes a scalar product of the two vectors. Here and afterwards the vector quantities are shown by bold symbols.

In the simplest case of a $\delta$-correlated canonical bath, the non-Markovian kinetic Eqs. (14)-(16) reduce to the standard Fokker-Planck equation (FPE)

$$
\begin{aligned}
\left(\frac{\partial}{\partial t}\right. & \left.+\frac{\mathbf{p}}{m} \cdot \boldsymbol{\nabla}_{\mathbf{r}}-\nabla_{\mathbf{r}} u(\mathbf{r}) \cdot \nabla_{\mathbf{p}}\right) f(\mathbf{p}, \mathbf{r} ; t) \\
& =\xi m T \boldsymbol{\nabla}_{\mathbf{p}} \cdot\left(\boldsymbol{\nabla}_{\mathbf{p}}+\frac{\mathbf{p}}{m T}\right) f(\mathbf{p}, \mathbf{r} ; t),
\end{aligned}
$$

where $\xi$ is the friction constant, see Appendix A. Note, that the $\delta$-correlated bath corresponds to the model of Brownian motion (heavy particle immersed in a bath of light ones), where the time scales of the particle motion and of the reorganization of the bath are strongly separated. In reality, however, the bath has a short, but finite characteristic correlation time $\tau_{c}$. The analysis of the associated effects is done in the following section.

\section{NON-MARKOVIAN EFFECTS IN THE MOMENTUM RELAXATION}

In order to simplify the analysis of non-Markovian effects, we set $u(\mathbf{r})=0$, and consider the process of relaxation in momentum space. Evolution of the distribution $f(\mathbf{p} ; t)$ can alternatively be characterized by considering the evolution of the probability momenta

$$
\begin{aligned}
& \mathbf{p}_{n}(t)=\int \mathbf{p}^{n} f(\mathbf{p} ; t) d \mathbf{p}, \quad \widetilde{\mathbf{p}}_{n}(\omega)=\int_{-\infty}^{\infty} \mathbf{p}_{n}(t) e^{-i \omega t} d t, \\
& n=1,2, \ldots,
\end{aligned}
$$

where tilde denotes the Fourier transform. Then we derive the following equation for the Fourier transform of the average momentum:

$$
\widetilde{\mathbf{p}}(\omega)=\frac{\mathbf{p}_{0}}{i \omega+\xi_{n}(\omega)},
$$

where

$$
\xi_{n}(\omega)=\left(1+\omega \frac{d}{d \omega}\right) \frac{\tilde{\mathcal{J}}(\omega)}{m T}
$$

is the nonstationary or frequency-dependent friction. Here

$$
\widetilde{\mathcal{J}}(\omega)=\int_{-\infty}^{\infty} \overline{\mathcal{J}}(t) e^{-i \omega t} d t
$$

is the Fourier transform of the force-force correlation function (A19).

The kinetics in the non-Markovian regime is sensitive to the functional form of the correlation function, and, for the sake of simplicity, we take its monoexponential form, given by Eq. (A23). This leads to

$$
\frac{\widetilde{\mathcal{J}}(\omega)}{m T}=\frac{\xi}{1+i \omega \tau_{c}},
$$

and

$$
\xi_{n}(\omega)=\frac{\xi}{\left(1+i \omega \tau_{c}\right)^{2}} .
$$

Reconstruction of the kinetics of the average momentum in the time domain calls for the solution of a cubic equation, which is of rather cumbersome form. Therefore, we give expressions for several limiting cases, depending on the value of the characteristic parameter $\xi \tau_{c}$. First of all, expanding the roots of the cubic equation, we obtain in the Markovian limit

$$
\mathbf{p}(t) \simeq \mathbf{p}_{0}\left(1+2 \xi \tau_{c}\right) e^{-\xi\left(1+2 \xi \tau_{c}\right) t}, \quad \xi \tau_{c} \ll 4 / 27, \quad t \gg \tau_{c} .
$$

Figure 2 shows the kinetics for the component of the average momentum at $\xi \tau_{c}=0.02$, calculated both from Eq. (43) (dashed line) and via the inverse Fourier transform of Eq. 


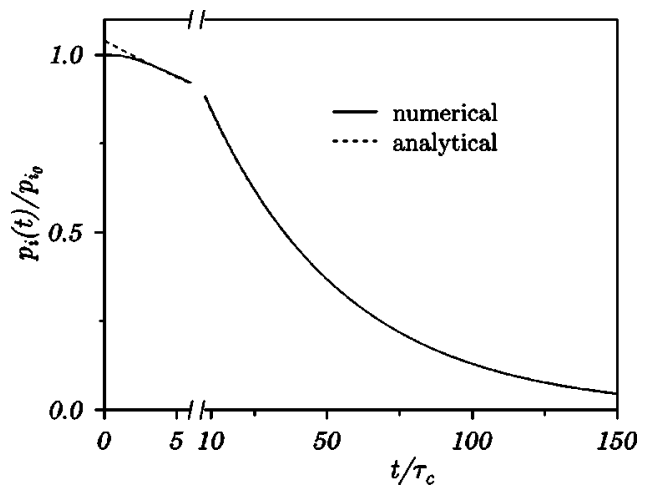

FIG. 2. Kinetics of the average momentum relaxation (for its component, $i=x, y$, or $z$ ) under conditions of the applicability of the Markovian limit, $\xi \tau_{c}=0.02$. The numerical result is obtained via the inverse Fourier transform of Eq. (38) and is plotted by the solid line. Analytic result of the Markovian limit, Eq. (43) is shown by the dashed line.

(38) (solid line). Some deviation between the results appears on the time scale of $\tau_{c}$, while at longer times the kinetics is accurately reproduced by Eq. (43). It is seen, that at short times the average momentum relaxes slower than exponentially, but proper modification of the initial conditions for the Markovian limit eliminates the difference for longer times.

Increasing the value of the characteristic parameter we arrive to the situation where $\xi \tau_{c}=4 / 27$, when the exact kinetics takes simple form

$$
\mathbf{p}(t) \simeq \mathbf{p}_{0} \frac{e^{-t /\left(3 \tau_{c}\right)}}{9}\left(8+4 t / \tau_{c}+e^{-t / \tau_{c}}\right), \quad \xi \tau_{c}=4 / 27
$$

At even larger values of $\xi \tau_{c}$ an oscillating contribution appears. Figure 3 shows the evolutions of the average momentum for $\xi \tau_{c}=4 / 27, \xi \tau_{c}=1 / 2$, and $\xi \tau_{c}=1$. It is seen, that the frequency of oscillations increases when increasing the friction. The origin of these oscillations can be explained by noting, that at finite reorganization time of the bath of $\sim \tau_{c}$ and sufficiently large interaction force the scattering on bath molecules is essentially elastic, and the particle is dynamically reflected several times before it completely loses the initial momentum. We pay heed to the restriction (A24) on the value of the friction due to the employed linear expan-

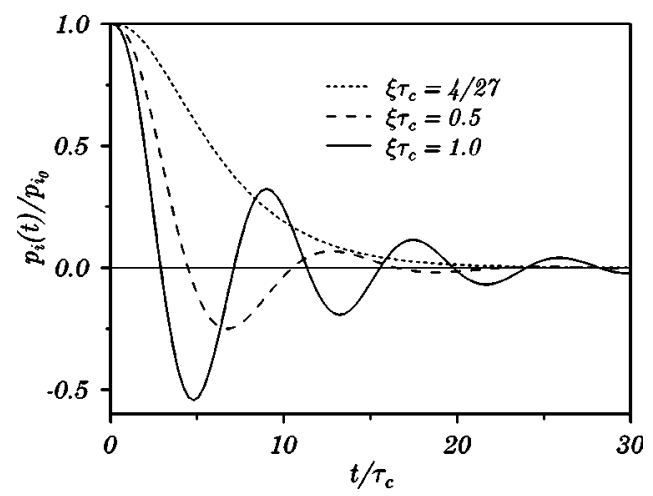

FIG. 3. Kinetics of the relaxation of average momentum component for $\xi \tau_{c}=4 / 27$ (dotted line), $\xi \tau_{c}=0.5$ (dashed line), and $\xi \tau_{c}=1.0$ (solid line). The numerical result were obtained via the inverse Fourier transform of Eq. (38).

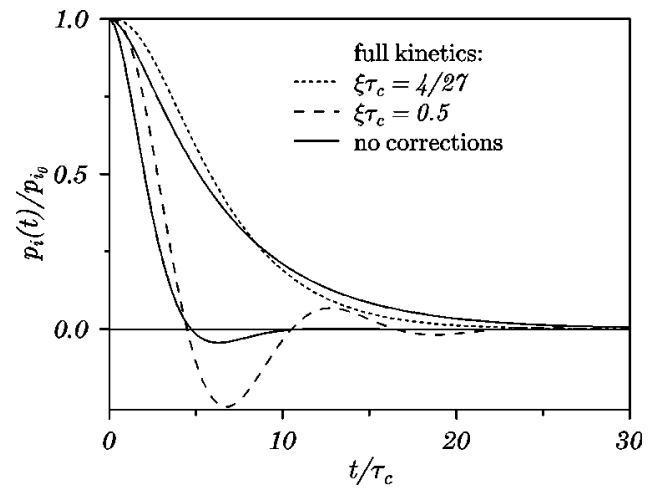

FIG. 4. Effect of quantum corrections on the kinetics of non-Markovian relaxation of average momentum. Full kinetics is calculated for $\xi \tau_{c}=4 / 27$ (dotted line), $\xi \tau_{c}=0.5$ (dashed line), while the corresponding kinetics neglecting quantum corrections are plotted by solid lines.

sion of the interaction potential, while the use of realistic (nonexpanded) interaction potentials requires more general non-Markovian kinetic Eqs. (14)-(16).

As it was stated above, quantum corrections may significantly affect the non-Markovian part of the kinetics. Indeed, neglecting the corresponding term in Eq. (39) (it contains derivative), we obtain Eq. (38), but with different nonstationary friction $\xi_{n}(\omega)=\xi /\left(1+i \omega \tau_{c}\right)$. Both forms of $\xi_{n}(\omega)$ yield asymptotically identical results for the Markovian limit $\left(\xi \tau_{c} \ll 1, t \gg \tau_{c}\right.$ ), but produce considerable deviations if the relaxation is of non-Markovian character, see Fig. 4.

Similar effects may be observed in the kinetics of the energy relaxation, and we briefly present the corresponding results. The Fourier transform of the second probability momentum (scalar value) is

$$
\tilde{p}_{2}(\omega)=\frac{p_{2_{0}}+6 \tilde{\mathcal{J}}(\omega) /(i \omega)}{i \omega+2 \xi_{n}(\omega)}
$$

where $\xi_{n}(\omega)$ and $\widetilde{\mathcal{J}}(\omega)$ were defined earlier, see Eqs. (39) and (40). Note, that starting from the second probability momentum the quantum corrections do not simply change the form of the non-stationary friction, but also affect the structure of the solution in the Fourier domain. Using Eqs. (41) and (42) we obtain, that the Markovian limit requires $\xi \tau_{c}$ $\ll 2 / 27$ and $t \gg \tau_{c}$, which yields exponential kinetics for the relaxation to the nonzero equilibrium value of $3 m T$ with the characteristic rate constant

$$
k_{2}=2 \xi\left(1+4 \xi \tau_{c}\right),
$$

while for $\xi \tau_{c}>2 / 27$ the kinetics gets an oscillatory contribution.

\section{DIFFUSION IN POSITION SPACE}

We now turn to the case of nonzero potential of the external forces $u(\mathbf{r})$ and obtain kinetic equations for long times, exceeding the time scale of momentum relaxation. The change to the reduced description by means of diffusion in the position space is possible in the so-called overdamped limit, when the potential of external forces does not change significantly the equilibrium distribution in momentum space. 
Traditionally, a so-called adiabatic elimination of the momentum is carried out in the framework of the Langevin approach, which is equivalent to FPE. However, in the case of the partial classical limit, the kinetic equations of the method contain quantum variables as well, and the corresponding equivalent stochastic differential equations can hardly be obtained. Therefore, we propose a simple alternative procedure to obtain the Smoluchowski equation starting directly from FPE, which can be extended to more complicated situations, as mentioned above. Besides, the presented technique allows to account for the modification of initial conditions during the preliminary stage of the relaxation of initial momentum.

The mathematical derivation of the Smoluchowski equation is given in Appendix B, which yields for the RDF in coordinate space $g(\mathbf{r} ; t)$ the following kinetic equation:

$$
\frac{\partial g(\mathbf{r} ; t)}{\partial t}=D \boldsymbol{\nabla}_{\mathbf{r}} \cdot\left(\boldsymbol{\nabla}_{\mathbf{r}} g(\mathbf{r} ; t)+g(\mathbf{r} ; t) \frac{\boldsymbol{\nabla}_{\mathbf{r}} u(\mathbf{r})}{T}\right),
$$

with the modified initial condition

$$
\begin{aligned}
g(\mathbf{r} ; 0) & =\int e^{-\nabla_{\mathbf{r}} \cdot \mathbf{p} /(\xi m)} f_{0}(\mathbf{r}, \mathbf{p}) d \mathbf{p} \\
& =\int f_{0}[\mathbf{r}-\mathbf{p} /(\xi m), \mathbf{p}] d \mathbf{p} .
\end{aligned}
$$

Here $D$ is the usual diffusion coefficient in the position space

$$
D=\frac{T}{\xi m} \text {. }
$$

The modified initial condition (48) accounts for the transformation of the initial distribution during the preliminary stage of momentum relaxation. This effect may be important for the geminate recombination in the condensed phase, if the pair of reactants originates from a highly excited parent molecule. In the case of a simple initial distribution of the form

$$
f_{0}(\mathbf{r}, \mathbf{p})=g_{0}(\mathbf{r}) \delta\left(\mathbf{p}-\mathbf{p}_{0}\right),
$$

we obtain from Eq. (48) that

$$
g(\mathbf{r} ; 0)=g_{0}\left[\mathbf{r}-\mathbf{p}_{0} /(\xi m)\right],
$$

i.e., the initial distribution is shifted. The value of the shift is determined by the initial velocity multiplied by the characteristic time $1 / \xi$ of relaxation in momentum space.

\section{CONCLUSION}

We presented the full classical limit of the recently proposed quantum-classical approximation. ${ }^{25}$ The obtained kinetic equations for the reduced distribution function in phase space have wide applicability limits and are capable of describing the non-Markovian evolution of an open system coupled to a canonical bath. They incorporate detailed balance directly, rather than through the use of the fluctuationdissipation theorem, and are valid beyond the linear response regime. The presented form of the kinetic equations allows to use arbitrary canonically conjugate coordinates in phase space.

The original quantum-classical treatment is based on a short coherence time $\tau_{b}$ of cross correlations between quan- tum subsystem and canonical bath due to the energy dispersion of bath degrees of freedom. This underlying physical process is of quantum-mechanical nature and is eliminated from the explicit consideration when passing to the classical limit. Nonetheless, the specific correlations between the open system and the bath, created during the neglected timescale of $\tau_{b}$, enter the classical limit via the modified initial conditions and the projected dynamics. In other words, the classical mechanics treatment of the open system is allowed only in the particular subspace of correlations, while the decay of correlations in the complementary subspace is essentially of quantum-mechanical nature and, therefore, cannot be correctly described classically. We have shown, that the projection (quantum correction) may significantly affect the nonMarkovian kinetics, and is required to preserve positivity of the reduced distribution function within the applicability limits of the approach.

Non-Markovian effects in the dynamics of open system are generally important on the time scale, comparable or shorter than the characteristic correlation time of the bath. On the other hand, the long time behavior is well described by considerably simplified kinetic equations, and their form is known for many cases of interest. Needless to say, that the obtained equations must correctly reproduce corresponding asymptotic behavior. Using the simple model of a heavy particle in an external field coupled to a bath of light solvent molecules, we obtained in the Markovian limit the standard Fokker-Planck equation. This example cannot serve as a proof for the general case, but we believe that the nonMarkovian equations of the classical limit provide the correct description of the kinetics on the entire time axis for all other systems, where the applicability criterion of the approach is fulfilled.

Although nonequilibrium molecular dynamics simulations seem to be a more natural way to study the nonMarkovian evolution of open systems in the classical limit, they do not account for quantum corrections in the short-time dynamics, and do not allow for a consistent incorporation of quantum transitions. On the other hand, the recently suggested quantum-classical approximation ${ }^{25}$ treats open system quantum mechanically, which can be prohibitively expensive for reactive systems with multidimensional reaction coordinates and/or small splittings between energy levels (rotational motion at high temperatures, etc.). The problem may frequently be overcome by taking the partial classical limit for several degrees of freedom of the open system, keeping a quantum-mechanical treatment for the most important ones. This can hardly be done applying partial Wigner transforms directly to the quantum Liouville-von Neumann equation for the density matrix of the total system as the resulting quantum-classical Liouville equation suffers from internal inconsistencies (see Introduction for more details), but seems to be permissible in the framework of the novel quantumclassical approach. ${ }^{25}$ The corresponding kinetic equations, combining classical mechanics description of the motion along the reaction coordinate and a nonphenomenological treatment of quantum transitions between them will be obtained and analyzed in the subsequent articles. 


\section{ACKNOWLEDGMENTS}

The author is grateful to Professor Jörg Schroeder and Professor Dirk Schwarzer for helpful discussions, and to Professor Jürgen Troe for his continuous support.

\section{APPENDIX A: REDUCTION TO FOKKER-PLANCK EQUATION}

We introduce generalized variables for the arbitrary function $a(t)$ using the stepwise Heaviside $\theta$ function as

$$
\bar{a}(t)=\theta(t) a(t) .
$$

In the following the corresponding generalized variables will be denoted by the bar accent. Then, Eqs. (14)-(16), and (34) give

$$
\begin{aligned}
\frac{\partial \bar{f}(\mathbf{p}, \mathbf{r} ; t)}{\partial t}= & \delta(t) f_{0}(\mathbf{p}, \mathbf{r})+\mathcal{A} \bar{f}(\mathbf{p}, \mathbf{r} ; t) \\
- & \int \mathbf{F}(\mathbf{r}, q) \cdot \boldsymbol{\nabla}_{\mathbf{p}} \bar{\eta}(\mathbf{p}, \mathbf{r}, q ; t) d q, \\
\frac{\partial \bar{\eta}(\mathbf{p}, \mathbf{r}, q ; t)}{\partial t}= & \left(\mathcal{A}+\mathcal{L}_{q}\right) \bar{\eta}(\mathbf{p}, \mathbf{r}, q ; t)-\mathbf{F}(\mathbf{r}, q) \\
& \cdot\left(\boldsymbol{\nabla}_{\mathbf{p}}+\frac{\mathbf{p}}{m T}\right) \bar{f}(\mathbf{p}, \mathbf{r} ; t) \\
& +\frac{W(\mathbf{r}, q)}{T}\left(\frac{\partial}{\partial t}-\mathcal{A}\right) \bar{f}(\mathbf{p}, \mathbf{r} ; t),
\end{aligned}
$$

where we have defined differential operator

$$
\mathcal{A}=-\frac{\mathbf{p}}{m} \cdot \nabla_{\mathbf{r}}+\left[\nabla_{\mathbf{r}} u(\mathbf{r})\right] \cdot \nabla_{\mathbf{p}},
$$

and vector of the forces

$$
\mathbf{F}(\mathbf{r}, q) \equiv-\nabla_{\mathbf{r}} W(\mathbf{r}, q),
$$

acting on the particle from the bath.

The formal solution of Eq. (A3) is expressed in terms of the free Green's function

$$
\overline{\mathcal{G}}_{0}\left(\mathbf{p}, \mathbf{r}, q \mid \mathbf{p}_{0}, \mathbf{r}_{0}, q_{0} ; t\right)=\bar{G}_{0}\left(\mathbf{p}, \mathbf{r} \mid \mathbf{p}_{0}, \mathbf{r}_{0} ; t\right) \bar{G}_{b}\left(q \mid q_{0} ; t\right),
$$

describing the evolution of the open system and the bath in the absence of their interaction. Here the free Green's function of open system obeys

$$
\left(\frac{\partial}{\partial t}-\mathcal{A}\right) \bar{G}_{0}\left(\mathbf{p}, \mathbf{r} \mid \mathbf{p}_{0}, \mathbf{r}_{0} ; t\right)=\delta(t) \delta\left(\mathbf{p}-\mathbf{p}_{0}\right) \delta\left(\mathbf{r}-\mathbf{r}_{0}\right),
$$

while the Green's function of the bath satisfy

$$
\left(\frac{\partial}{\partial t}-\mathcal{L}_{q}\right) \bar{G}_{b}\left(q \mid q_{0} ; t\right)=\delta(t) \delta\left(q-q_{0}\right) .
$$

The solution of Eq. (A7) is readily obtained, and corresponds to the motion of particle along the classical trajectory, specified by the Hamiltonian (34)

$$
\begin{aligned}
\bar{G}_{0}\left(\mathbf{p}, \mathbf{r} \mid \mathbf{p}_{0}, \mathbf{r}_{0} ; t\right)= & \theta(t) \delta\left[\mathbf{p}-\mathbf{p}_{t}\left(\mathbf{p}_{0}, \mathbf{r}_{0} ; t\right)\right] \\
& \times \delta\left[\mathbf{r}-\mathbf{r}_{t}\left(\mathbf{p}_{0}, \mathbf{r}_{0} ; t\right)\right]
\end{aligned}
$$

where $\mathbf{p}_{t}\left(\mathbf{p}_{0}, \mathbf{r}_{0} ; t\right)$ and $\mathbf{r}_{t}\left(\mathbf{p}_{0}, \mathbf{r}_{0} ; t\right)$ are the phase space coordinates, where the open system arrives after time $t$, starting from the coordinates $\left(\mathbf{p}_{0}, \mathbf{r}_{0}\right)$. The free dynamics (without interaction with the bath) is reversible, and, therefore, Eq. (A9) can also be written as

$$
\begin{aligned}
& \bar{G}_{0}\left(\mathbf{p}, \mathbf{r} \mid \mathbf{p}_{0}, \mathbf{r}_{0} ; t\right) \\
& \quad=\theta(t) \delta\left[\mathbf{p}_{0}-\mathbf{p}_{t}(\mathbf{p}, \mathbf{r} ;-t)\right] \delta\left[\mathbf{r}_{0}-\mathbf{r}_{t}(\mathbf{p}, \mathbf{r} ;-t)\right] .
\end{aligned}
$$

Using Eqs. (A6) and (A10) we rewrite Eq. (A3) as fully equivalent integrodifferential one

$$
\begin{aligned}
\bar{\eta}(\mathbf{p}, \mathbf{r}, q ; t)= & \frac{1}{T} \iint_{-\infty}^{\infty} \bar{G}_{b}\left(q \mid q_{0} ; \tau\right) W\left(\mathbf{r}_{0}, q_{0}\right) \\
& \times\left(\frac{\partial}{\partial t}-\mathcal{A}_{0}\right) \bar{f}\left(\mathbf{p}_{0}, \mathbf{r}_{0} ; t-\tau\right) d q_{0} d \tau \\
& -\iint_{-\infty}^{\infty} \bar{G}_{b}\left(q \mid q_{0} ; \tau\right) \mathbf{F}\left(\mathbf{r}_{0}, q_{0}\right) \\
& \cdot\left[\boldsymbol{\nabla}_{\mathbf{p}_{0}}+\frac{\mathbf{p}_{0}}{m T}\right] \bar{f}\left(\mathbf{p}_{0}, \mathbf{r}_{0} ; t-\tau\right) d q_{0} d \tau,
\end{aligned}
$$

where $\mathbf{p}_{0}=\mathbf{p}_{t}(\mathbf{p}, \mathbf{r} ;-\tau), \mathbf{r}_{0}=\mathbf{r}_{t}(\mathbf{p}, \mathbf{r} ;-\tau)$, and

$$
\mathcal{A}_{0}=-\frac{\mathbf{p}_{0}}{m} \cdot \boldsymbol{\nabla}_{\mathbf{r}_{0}}+\left[\boldsymbol{\nabla}_{\mathbf{r}_{0}} u\left(\mathbf{r}_{0}\right)\right] \cdot \boldsymbol{\nabla}_{\mathbf{p}_{0}} .
$$

For short times these sets of phase space coordinates are related to each other by

$$
\mathbf{p}_{0} \simeq \mathbf{p}+\tau \nabla_{\mathbf{r}} u(\mathbf{r}), \quad \mathbf{r}_{0} \simeq \mathbf{r}-\tau \mathbf{p} / m .
$$

Substituting Eq. (A11) in the right hand side of Eq. (A2), we get the fully equivalent integrodifferential equation for the RDF of the open system

$$
\begin{aligned}
\frac{\partial \bar{f}(\mathbf{p}, \mathbf{r} ; t)}{\partial t}= & \delta(t) f_{0}(\mathbf{p}, \mathbf{r})+\mathcal{A} \bar{f}(\mathbf{p}, \mathbf{r} ; t)-\frac{1}{T} \int_{-\infty}^{\infty} \boldsymbol{\nabla}_{\mathbf{p}} \\
& \cdot \mathbf{C}(\mathbf{p}, \mathbf{r} ; \tau)\left(\frac{\partial}{\partial t}-\mathcal{A}_{0}\right) \bar{f}\left(\mathbf{p}_{0}, \mathbf{r}_{0} ; t-\tau\right) d \tau \\
& +\int_{-\infty}^{\infty} \nabla_{\mathbf{p}} \cdot \hat{\mathbf{K}}(\mathbf{p}, \mathbf{r} ; \tau) \cdot\left(\boldsymbol{\nabla}_{\mathbf{p}_{0}}+\frac{\mathbf{p}_{0}}{m T}\right) \\
& \times \bar{f}\left(\mathbf{p}_{0}, \mathbf{r}_{0} ; t-\tau\right) d \tau .
\end{aligned}
$$

Here the vector

$$
\mathbf{C}(\mathbf{p}, \mathbf{r} ; \tau)=\iint \mathbf{F}(\mathbf{r}, q) \bar{G}_{b}\left(q \mid q_{0} ; \tau\right) W\left(\mathbf{r}_{0}, q_{0}\right) d q d q_{0}
$$

specifies correlations between the force and the shifted potential, while elements of the tensor

$$
\begin{aligned}
K_{i j}(\mathbf{p}, \mathbf{r} ; \tau)= & \iint F_{i}(\mathbf{r}, q) \bar{G}_{b}\left(q \mid q_{0} ; \tau\right) F_{j}\left(\mathbf{r}_{0}, q_{0}\right) d q d q_{0}, \\
& i, j=x, y, z
\end{aligned}
$$

contain information about correlations between components of the shifted forces. 
The structure of the correlation functions (A15) and (A16) depends on the properties of the bath and of the system-bath coupling. Although it is not difficult to continue with the more general case of an anisotropic bath, we assume, for the clarity of presentation, that the bath is isotropic. In this case $\mathbf{C}(\mathbf{p}, \mathbf{r} ; \tau)=\mathbf{C}(\mathbf{p} ; \tau)$, and $K_{i j}(\mathbf{p}, \mathbf{r} ; \tau)$ $=K_{i j}(\mathbf{p} ; \tau)$, i.e., the correlations do not depend on the position of the open system.

The subsequent simplification of the structure of the correlation functions (A15) and (A16) is possible only when assuming a sufficiently short correlation time $\tau_{c}$ of the bath to expand the shifted interaction potential as

$$
W\left(\mathbf{r}_{0}, q_{0}\right) \simeq W\left(\mathbf{r}, q_{0}\right)+\tau \frac{\mathbf{p}}{m} \cdot \mathbf{F}\left(\mathbf{r}, q_{0}\right) .
$$

Due to symmetry reasons the first term of expansion (A17) does not give contributions to Eq. (A15), while the different components of the force are uncorrelated. A similar expansion can be written for the shifted force, but then the corresponding linear term on $\tau$ does not give contributions to Eq. (A16) (symmetry reasons again). After that Eqs. (A15) and (A16) are simplified to

$$
\mathbf{C}(\mathbf{p} ; \tau) \simeq \frac{\mathbf{p}}{m} \tau \overline{\mathcal{J}}(\tau), \quad \hat{\mathbf{K}}(\mathbf{p} ; \tau) \simeq \hat{\mathbf{1}} \overline{\mathcal{J}}(\tau),
$$

where $\hat{\mathbf{1}}$ is the unity tensor, while the correlation function of the bath

$$
\begin{gathered}
\overline{\mathcal{J}}(\tau)=\iint F_{i}(\mathbf{r}, q) \bar{G}_{b}\left(q \mid q_{0} ; \tau\right) F_{i}\left(\mathbf{r}, q_{0}\right) d q d q_{0}, \\
i=x, y, \quad \text { or } z .
\end{gathered}
$$

For long times, the dynamical properties of the bath are usually specified by the friction $\xi$, which is expressed via the force-force correlation function (A19) as

$$
\xi=\frac{1}{m T} \int_{-\infty}^{\infty} \overline{\mathcal{J}}(\tau) d \tau
$$

In the simplest situation of a $\delta$-correlated bath, when

$$
\overline{\mathcal{J}}(\tau)=\xi m T \delta(\tau),
$$

Eqs. (A14) and (A18) yield the standard Fokker-Planck Eq. (36).

Nevertheless, the bath has a short, but finite correlation time $\tau_{c}$, and at large frictions $\left(\xi \tau_{c} \gtrsim 1\right)$ relaxation in the momentum space has strongly non-Markovian character. The kinetics in this regime is sensitive to the form of the correlation function (A19), while the short time expansion of the shifted potential (A17) becomes of limited use. Indeed, estimating absolute value of the momentum as $\left|p_{i}\right| \sim \sqrt{2 m E_{k}}$, where $E_{k}$ is the kinetic energy of the solute, Eqs. (19) and (A17) lead to the following restriction on the value of the interaction force

$$
|F| \ll \frac{\pi m T}{\tau_{c} \sqrt{2 m E_{k}}} .
$$

In turn, the value of the friction for the exponential form of the correlation function

$$
\overline{\mathcal{J}}(\tau)=\theta(\tau) F^{2} e^{-\tau / \tau_{c}}, \quad \xi=F^{2} \tau_{c} /(m T),
$$

which gives

$$
\xi \tau_{c} \sim \frac{F^{2} \tau_{c}^{2}}{m T} \ll \frac{\pi^{2} T}{2 E_{k}} .
$$

In other words, the validity of the linear expansion (A17) of the shifted potential on the time scale of the correlation time depends on the kinetic energy of the solute. Near equilibrium $E_{k} \sim T$, and at $\xi \tau_{c} \gtrsim 1$ Eq. (A17) cannot be justified. The restriction on the allowed frictions $\xi \tau_{c} \lesssim 1$ is more severe, if the initial kinetic energy of the solute considerably exceeds its thermal value. On the other hand, the rejection of the linear expansion (A17) leads to a nonlinear dependence of the memory kernels on momentum. In that case we come back to the more general non-Markovian equations of Sec. III.

In a simple situation, when the potential of external force is absent $[u(\mathbf{r})=0]$, the non-Markovian FPE, suitable to analyze the effects of finite correlation time at not too strong frictions $\left(\xi \tau_{c} \lesssim 1\right)$ is readily obtained. In that case one has $\mathbf{p}_{0}=\mathbf{p}$, since the free evolution of the open system is a rectilinear motion with a constant velocity. Then, taking account for Eq. (A18), and integrating both sides of Eq. (A14) on coordinates, we obtain for the RDF in the momentum space

$$
\bar{f}(\mathbf{p} ; t)=\int \bar{f}(\mathbf{p}, \mathbf{r} ; t) d \mathbf{r}, \quad f_{0}(\mathbf{p})=\int f_{0}(\mathbf{p}, \mathbf{r}) d \mathbf{r}
$$

the following kinetic equation:

$$
\begin{aligned}
\frac{\partial \bar{f}(\mathbf{p} ; t)}{\partial t}= & \delta(t) f_{0}(\mathbf{p}) \\
& -\frac{1}{m T} \int_{-\infty}^{\infty} \tau \overline{\mathcal{J}}(\tau) \boldsymbol{\nabla}_{\mathbf{p}} \cdot \mathbf{p} \frac{\partial \bar{f}(\mathbf{p} ; t-\tau)}{\partial t} d \tau \\
& +\int_{-\infty}^{\infty} \overline{\mathcal{J}}(\tau) \boldsymbol{\nabla}_{\mathbf{p}} \cdot\left(\boldsymbol{\nabla}_{\mathbf{p}}+\frac{\mathbf{p}}{m T}\right) \bar{f}(\mathbf{p} ; t-\tau) d \tau .
\end{aligned}
$$

Note, that Eq. (A26) should be applied with care, since it may violate positivity at far tails of the distribution, because in the situation of finite correlation time there exists some finite value of the momentum, which already breaks down the linear expansion (A17). This is removed in the limit $\tau_{c}$ $\rightarrow 0$, so that the Fokker-Planck equation does preserve positivity. Therefore, we prefer using Eq. (A26) to obtain nonMarkovian kinetic equations for several low-order probability momenta (37), for which the above problem does not appear.

\section{APPENDIX B: DERIVATION OF THE SMOLUCHOWSKI EQUATION}

Assuming separation of time scales for the relaxation in momentum and coordinate spaces, one can derive the equation for the reduced distribution function in the coordinate space, which is defined as 


$$
\bar{g}(\mathbf{r} ; t)=\int \bar{f}(\mathbf{r}, \mathbf{p} ; t) d \mathbf{p}, \quad g_{0}(\mathbf{r})=\int f_{0}(\mathbf{r}, \mathbf{p}) d \mathbf{p} .
$$

Then, integrating Eq. (36) over momentum variables, we obtain

$$
\frac{\partial \bar{g}(\mathbf{r} ; t)}{\partial t}=\delta(t) g_{0}(\mathbf{r})-\frac{\bar{\gamma}_{1}(\mathbf{r} ; t)}{m},
$$

where

$$
\begin{aligned}
\bar{\gamma}_{n}(\mathbf{r} ; t) & =\int\left(\boldsymbol{\nabla}_{\mathbf{r}} \cdot \mathbf{p}\right)^{n} \bar{f}(\mathbf{r}, \mathbf{p} ; t) d \mathbf{p}, \\
\gamma_{n_{0}}(\mathbf{r}) & =\int\left(\nabla_{\mathbf{r}} \cdot \mathbf{p}\right)^{n} f_{0}(\mathbf{r}, \mathbf{p}) d \mathbf{p}, \quad n=1,2, \ldots .
\end{aligned}
$$

In turn, Eq. (36) yields

$$
\begin{aligned}
\frac{\partial \bar{\gamma}_{1}(\mathbf{r} ; t)}{\partial t}= & \delta(t) \gamma_{1_{0}}(\mathbf{r})-\xi \bar{\gamma}_{1}(\mathbf{r} ; t) \\
& -\left[\frac{\bar{\gamma}_{2}(\mathbf{r} ; t)}{m}+\boldsymbol{\nabla}_{\mathbf{r}} \cdot\left[\bar{g}(\mathbf{r} ; t) \nabla_{\mathbf{r}} u(\mathbf{r})\right]\right],
\end{aligned}
$$

where $\xi$ is the friction, see Eq. (A20). This hierarchy can be continued to give

$$
\begin{aligned}
\frac{\partial \bar{\gamma}_{n}(\mathbf{r} ; t)}{\partial t}= & \delta(t) \gamma_{n_{0}}(\mathbf{r})-\left[\frac{\bar{\gamma}_{n+1}(\mathbf{r} ; t)}{m}+\bar{\kappa}_{n}(\mathbf{r} ; t)\right] \\
& -n \xi\left[\bar{\gamma}_{n}(\mathbf{r} ; t)-m T(n-1) \boldsymbol{\nabla}_{\mathbf{r}} \cdot \nabla_{\mathbf{r}} \bar{\gamma}_{n-2}(\mathbf{r} ; t)\right],
\end{aligned}
$$

where

$$
\bar{\kappa}_{n}(\mathbf{r} ; t)=n \int\left(\boldsymbol{\nabla}_{\mathbf{r}} \cdot \mathbf{p}\right)^{n-1} \boldsymbol{\nabla}_{\mathbf{r}} \cdot\left[\bar{f}(\mathbf{r}, \mathbf{p} ; t) \boldsymbol{\nabla}_{\mathbf{r}} u(\mathbf{r})\right] d \mathbf{p} .
$$

The Smoluchowski equation follows from Eq. (B4) assuming that after time of $1 / \xi$, the momentum relaxes to its equilibrium value, while the potential of the external forces $u(\mathbf{r})$ does not significantly change the resulting Maxwellian velocity distribution (overdamped limit). In that case one can assume

$$
\bar{f}(\mathbf{r}, \mathbf{p} ; t) \simeq \frac{1}{(2 \pi m T)^{3 / 2}} \exp \left(-\frac{\mathbf{p}^{2}}{2 m T}\right) \bar{g}(\mathbf{r} ; t),
$$

for the calculation of $\bar{\gamma}_{2}(\mathbf{r} ; t)$ (closure approximation), which gives

$$
\bar{\gamma}_{2}(\mathbf{r} ; t) \simeq m T \nabla_{\mathbf{r}} \cdot \nabla_{\mathbf{r}} \bar{g}(\mathbf{r} ; t) .
$$

Then, the quasistationary solution of Eq. (B4) at times $t$ $\gg 1 / \xi$ is

$\bar{\gamma}_{1}(\mathbf{r} ; t) \simeq \delta(t) \gamma_{1_{0}}^{\prime}(\mathbf{r})-\frac{T}{\xi} \nabla_{\mathbf{r}} \cdot\left(\nabla_{\mathbf{r}} \bar{g}(\mathbf{r} ; t)+\bar{g}(\mathbf{r} ; t) \frac{\boldsymbol{\nabla}_{\mathbf{r}} u(\mathbf{r})}{T}\right)$,

which, upon substitution in Eq. (B2) and changing to nongeneralized variables, yields Eqs. (47)-(49). Here the modified initial condition

$$
\gamma_{1_{0}}^{\prime}(\mathbf{r})=\sum_{n=1}^{\infty} \int \frac{(-1)^{n}}{n ! \xi^{n} m^{n-1}}\left(\boldsymbol{\nabla}_{\mathbf{r}} \cdot \mathbf{p}\right)^{n} f_{0}(\mathbf{r}, \mathbf{p}) d \mathbf{p} .
$$

Note, that Eq. (B10) have appeared as the result of partial summation of the contributions from the equations of higher order correlations $\bar{\gamma}_{n}(\mathbf{r} ; t)$, which gives in the quasistationary regime $(t \gg 1 / \xi)$

$$
\bar{\gamma}_{n}(\mathbf{r} ; t) \sim \delta(t) \frac{\gamma_{n_{0}}(\mathbf{r})}{n \xi}-\frac{\bar{\gamma}_{n+1}(\mathbf{r} ; t)}{n \xi m},
$$

and, taking into account Eq. (B3), leads to Eq. (B10). Such a partial summation is required to prevent possible violation of positivity of the modified initial distribution (48).

Equation (B5) can serve as the basis to derive systematic corrections to the Smoluchowski equation on the smallness of the inverse friction $1 / \xi$. This is achieved by considering more members of the hierarchy (B5), which allows to reproduce known results. ${ }^{34-36}$

${ }^{1}$ R. Kubo, J. Phys. Soc. Jpn. 12, 570 (1957)

${ }^{2}$ R. Kubo, M. Toda, and N. Hashitsume, Statistical Physics II: Nonequilibrium Statistical Mechanics (Springer, Berlin, 1978).

${ }^{3}$ L. D. Landau and E. M. Lifshitz, Statistical Physics, Course of Theoretical Physics (Pergamon, Oxford, 1980), Vol. 5.

${ }^{4}$ M. P. Allen and D. J. Tildesley, Computer Simulation of Liquids (Clarendon, Oxford, 1996).

${ }^{5}$ P. Ehrenfest, Z. Phys. 45, 455 (1927).

${ }^{6}$ H.-D. Meyer and W. H. Miller, J. Chem. Phys. 70, 3214 (1979).

${ }^{7}$ K. Drukker, J. Comput. Phys. 153, 225 (1999), and references therein.

${ }^{8}$ J. C. Tully, J. Chem. Phys. 93, 1061 (1990).

${ }^{9}$ S. Hammes-Schiffer and J. C. Tully, J. Chem. Phys. 101, 4657 (1994).

${ }^{10}$ O. V. Prezhdo and P. J. Rossky, J. Chem. Phys. 107, 825 (1997).

${ }^{11}$ E. Wigner, Phys. Rev. 40, 749 (1932).

${ }^{12}$ J. E. Moyal, Proc. Cambridge Philos. Soc. 45, 99 (1945).

${ }^{13}$ I. V. Alexandrov, Z. Naturforsch. A 36, 902 (1981).

${ }^{14}$ W. Boucher and J. Traschen, Phys. Rev. D 37, 3522 (1988).

${ }^{15}$ A. Anderson, Phys. Rev. Lett. 74, 621 (1995).

${ }^{16}$ O. V. Prezhdo and V. V. Kisil, Phys. Rev. A 56, 162 (1997).

${ }^{17}$ R. Kapral and G. Cicotti, J. Chem. Phys. 110, 8919 (1999).

${ }^{18}$ S. Nakajima, Prog. Theor. Phys. 20, 948 (1958); R. Zwanzig, J. Chem. Phys. 33, 1338 (1960)

${ }^{19}$ N. G. van Kampen, Stochastic Processes in Physics and Chemistry North-Holland, Amsterdam, 1981)

${ }^{20}$ Y. Tanimura and P. G. Wolynes, Phys. Rev. A 43, 4131 (1991); Y. Tanimura and P. G. Wolynes, J. Chem. Phys. 96, 8485 (1992).

${ }^{21}$ U. Weiss, Quantum Dissipative Systems (World Scientific, Singapore, 1993).

${ }^{22}$ K. Blum, Density Matrix Theory and Applications (Plenum, New York, 1995).

${ }^{23}$ C. Meier and D. J. Tannor, J. Chem. Phys. 111, 3365 (1999).

${ }^{24}$ J. Wilkie, J. Chem. Phys. 114, 7736 (2001).

${ }^{25}$ A. A. Neufeld, J. Chem. Phys. 119, 2488 (2003).

${ }^{26}$ A. A. Neufeld, D. Schwarzer, J. Schroeder, and J. Troe, J. Chem. Phys. 119, 2502 (2003).

${ }^{27}$ R. Zwanzig, Phys. Rev. 124, 983 (1961); R. Zwanzig, J. Stat. Phys. 9, 215 (1973).

${ }^{28}$ H. Mori, Prog. Theor. Phys. 33, 423 (1965).

${ }^{29}$ H. Risken, The Fokker-Planck Equation, Methods of Solution and Applications, 2nd ed. (Springer, Berlin, 1989), and references therein.

${ }^{30} \mathrm{R}$. Balescu, Equilibrium and Non Equilibrium Statistical Mechanics (Wiley, New York, 1975).

${ }^{31}$ P. Schofield, Phys. Rev. Lett. 4, 39 (1960).

${ }^{32}$ P. A. Egelstaff, Adv. Phys. 11, 203 (1962).

${ }^{33}$ B. J. Berne, J. Jortner, and R. Gordon, J. Chem. Phys. 47, 1600 (1967).

${ }^{34}$ P. A. Lee, J. Appl. Phys. 42, 325 (1971).

${ }^{35}$ G. Wilemski, J. Stat. Phys. 14, 153 (1976).

${ }^{36}$ U. M. Titulaer, Physica A 91, 321 (1978). 